\title{
Taylor theory associated with Hahn difference operator
}

Karima Oraby ${ }^{1 *}$ and Alaa Hamza ${ }^{2,3}$

"Correspondence: koraby83@yahoo.com

${ }^{1}$ Department of Mathematics and Computer Science, Faculty of Science, Suez University, Suez, Egypt Full list of author information is available at the end of the article

\section{Abstract}

In this paper, we establish Taylor theory based on Hahn's difference operator $D_{q, \omega}$ which is defined by $D_{q, \omega} f(t)=\frac{f(q t+\omega)-f(t)}{t(q-1)+\omega}, t \neq \frac{\omega}{1-q}$, where $q \in(0,1)$ and $\omega$ is a positive number.

MSC: 39A10; 39A13; 39A70; 41A58; 47B39

Keywords: Taylor series; Hahn difference operator $D_{q, \omega}$

\section{Introduction and preliminaries}

Let $q \in(0,1), \omega>0$ and $\omega_{0}:=\frac{\omega}{1-q}$. Let $f$ be a function defined on an interval $I$ of $\mathbb{R}$ which contains $\omega_{0}$. Hahn [10] introduced his difference operator which is defined by

$$
D_{q, \omega} f(t):=\frac{f(q t+\omega)-f(t)}{t(q-1)+\omega}, \quad \text { if } t \neq \omega_{0}
$$

and $D_{q, \omega} f\left(\omega_{0}\right):=f^{\prime}\left(\omega_{0}\right)$, provided that $f$ is differentiable at $\omega_{0}$ in the usual sense. In this case we call $D_{q, \omega} f$ the $q, \omega$-derivative and that $f$ is $q, \omega$-differentiable at $t$ whenever $D_{q, \omega} f(t)$ exists. Finally, we say that $f$ is $q, \omega$-differentiable, i.e., throughout $I$ if $D_{q, \omega} f\left(\omega_{0}\right)$ exists.

Hahn difference operator unifies the two most well-known quantum difference operators: the Jackson $q$-difference operator [11-13], which is defined by

$$
D_{q} f(t)=\frac{f(q t)-f(t)}{t(q-1)}, \quad \text { if } t \neq 0,0<q<1
$$

and the forward difference $\Delta_{\omega}$, which is defined by

$$
\Delta_{\omega} f(t)=\frac{f(t+\omega)-f(t)}{\omega}, \quad t \in \mathbb{R}, \omega>0,
$$

see $[4,5,14,15]$. Hahn operator has attracted the attention of several researchers and a variety of results can be found in papers [1, 2, 6, 16-22]. In [3] Annaby and Mansour proved analytically the $q$-Taylor series associated with $D_{q}$, introduced by Jackson [12], of an analytic function in some complex domain. In the present paper, we establish an overarching

(c) The Author(s) 2020. This article is licensed under a Creative Commons Attribution 4.0 International License, which permits use sharing, adaptation, distribution and reproduction in any medium or format, as long as you give appropriate credit to the original author(s) and the source, provide a link to the Creative Commons licence, and indicate if changes were made. The images or other third party material in this article are included in the article's Creative Commons licence, unless indicated otherwise in a credit line to the material. If material is not included in the article's Creative Commons licence and your intended use is not permitted by statutory regulation or exceeds the permitted use, you will need to obtain permission directly from the copyright holder. To view a copy of this licence, visit http://creativecommons.org/licenses/by/4.0/. 
$q, \omega$-Taylor theory associated with Hahn difference operator $D_{q, \omega}$. In this theory the Hahn difference operator $D_{q, \omega}$ replaces the differentiation operator in the usual Taylor series.

First, we introduce some preliminary results and some notations. Let $f, g$ be $q, \omega$ differentiable at $t \in I$, then

$$
\begin{aligned}
& D_{q, \omega}(f+g)(t)=D_{q, \omega} f(t)+D_{q, \omega} g(t), \\
& D_{q, \omega}(f g)(t)=D_{q, \omega}(f(t)) g(t)+f(q t+\omega) D_{q, \omega} g(t), \\
& D_{q, \omega}(f / g)(t)=\frac{D_{q, \omega}(f(t)) g(t)-f(t) D_{q, \omega} g(t)}{g(t) g(q t+\omega)}
\end{aligned}
$$

provided that in (1.6), $g(t) g(q t+\omega) \neq 0[1,2]$. Also, for $n \in \mathbb{N}$, the following relations hold:

$$
\begin{aligned}
& D_{q, \omega}(\alpha t+\beta)^{n}=\alpha \sum_{k=0}^{n-1}(\alpha(q t+\omega)+\beta)^{k}(\alpha t+\beta)^{n-k-1}, \\
& D_{q, \omega}(\alpha t+\beta)^{-n}=-\alpha \sum_{k=0}^{n-1}(\alpha(q t+\omega)+\beta)^{-n+k}(\alpha t+\beta)^{-k-1},
\end{aligned}
$$

where $\alpha, \beta \in \mathbb{R}$, see $[1,2]$.

The $q$-shifted factorial $(b ; q)_{n}$ for a complex number $b$ and $n \in \mathbb{N}_{0}=\mathbb{N} \cup\{0\}$ is defined to be

$$
(b ; q)_{n}= \begin{cases}\prod_{j=1}^{n}\left(1-b q^{j-1}\right), & \text { if } n \in \mathbb{N} \\ 1, & \text { if } n=0 .\end{cases}
$$

The limit $\lim _{n \rightarrow \infty}(b ; q)_{n}$ is denoted by $(b ; q)_{\infty}$. Moreover $(b ; q)_{n}$ has the representation [9]

$$
(b ; q)_{n}=\sum_{k=0}^{n}(-1)^{k}\left(\begin{array}{l}
n \\
k
\end{array}\right)_{q} q^{\frac{k(k-1)}{2}} b^{k} .
$$

The $q$-binomial coefficients [9]

$$
\left(\begin{array}{l}
n \\
k
\end{array}\right)_{q}=\frac{(q ; q)_{n}}{(q ; q)_{k}(q ; q)_{n-k}}
$$

satisfy the following property:

$$
\left(\begin{array}{c}
n+1 \\
k
\end{array}\right)_{q}=\left(\begin{array}{l}
n \\
k
\end{array}\right)_{q} q^{k}+\left(\begin{array}{c}
n \\
k-1
\end{array}\right)_{q}=\left(\begin{array}{l}
n \\
k
\end{array}\right)_{q}+\left(\begin{array}{c}
n \\
k-1
\end{array}\right)_{q} q^{n+1-k} .
$$

For $n \in \mathbb{N}_{0}$ and $0<q<1$, the $q$-analogues of the natural numbers of the factorial function and of the semifactorial function $[7,13]$ are defined by

$$
[n]_{q}=\frac{1-q^{n}}{1-q}, \quad n \in \mathbb{N}_{0}, 0<q<1,
$$


and

$$
[n]_{q} !=\prod_{k=1}^{n}[k]_{q}, \quad[0]_{q} !:=1, \quad 0<q<1
$$

$[x-a]_{n}$ is defined by

$$
[x-a]_{n}=(x-a)(x-a q)\left(x-a q^{2}\right) \cdots\left(x-a q^{n-1}\right), \quad n \geq 1, \quad[x-a]_{0}=1 .
$$

The following formula was obtained by Euler [8]:

$$
[x-a]_{n}=\sum_{k=0}^{n}\left(\begin{array}{l}
n \\
k
\end{array}\right)_{q} q^{\frac{k(k-1)}{2}} x^{n-k}(-a)^{k} .
$$

The q-gamma function [9] is defined by

$$
\Gamma_{q}(z)=\frac{(q ; q)_{\infty}}{\left(q^{z} ; q\right)_{\infty}}(1-q)^{1-z}, \quad 0<q<1,
$$

where $z \in \mathbb{C} \backslash\left\{-n: n \in \mathbb{N}_{0}\right\}$. Here, we take the principal values of $q^{z}$ and $(1-q)^{1-z}$. In particular

$$
\Gamma_{q}(n+1)=\frac{(q ; q)_{n}}{(1-q)^{n}}, \quad n \in \mathbb{N}
$$

It is known that, for $x>0, \Gamma_{q}(x)$ is the unique logarithmically convex function that satisfies the functional equation:

$$
\Gamma_{q}(x+1)=[x]_{q} \Gamma_{q}(x), \quad \Gamma_{q}(1)=1 .
$$

In [1], Aldowah introduced the $q, \omega$-integral of $f$ from $a$ to $b$ as follows.

Definition 1.1 Let $I$ be any interval of $\mathbb{R}$ containing $\omega_{0}$. Assume that $f: I \rightarrow \mathbb{R}$ is a function, and let $a, b \in I$ such that $a<b$. The $q, \omega$-integral of $f$ from $a$ to $b$ is defined by

$$
\int_{a}^{b} f(t) d_{q, \omega} t:=\int_{\omega_{0}}^{b} f(t) d_{q, \omega} t-\int_{\omega_{0}}^{a} f(t) d_{q, \omega} t
$$

where

$$
\int_{\omega_{0}}^{x} f(t) d_{q, \omega} t:=(x(1-q)-\omega) \sum_{k=0}^{\infty} q^{k} f\left(x q^{k}+\omega[k]_{q}\right), \quad x \in I,
$$

provided that the series converges at $x=a$ and $x=b$. In this case $f$ is called $q, \omega$-integrable over $[a, b]$ for all $a, b \in I$.

Lemma $1.2([1,2])$ Let $f, g: I \rightarrow \mathbb{R}$ be $q$, w-integrable on $I, k \in \mathbb{R}$ and $a, b, c \in I, a<c<b$. 
(i) $\int_{a}^{a} f(t) d_{q, \omega} t=0$,

(ii) $\int_{a}^{b} k f(t) d_{q, \omega} t=k \int_{a}^{b} f(t) d_{q, \omega} t$,

(iii) $\int_{a}^{b} f(t) d_{q, \omega} t=-\int_{b}^{a} f(t) d_{q, \omega} t$,

(iv) $\int_{a}^{b} f(t) d_{q, \omega} t=\int_{a}^{c} f(t) d_{q, \omega} t+\int_{c}^{b} f(t) d_{q, \omega} t$,

(v) $\int_{a}^{b}(f(t)+g(t)) d_{q, \omega} t=\int_{a}^{b} f(t) d_{q, \omega} t+\int_{a}^{b} g(t) d_{q, \omega} t$.

Lemma $1.3([1,2])$ If $f: I \rightarrow \mathbb{R}$ is continuous at $\omega_{0}$, then $\left\{f\left(s q^{k}+\omega[k]_{q}\right)\right\}_{k \in \mathbb{N}}$ converges uniformly to $f\left(\omega_{0}\right)$ on $I$.

Corollary 1.4 ([1,2]) Iff $: I \rightarrow \mathbb{R}$ is continuous at $\omega_{0}$, then $\sum_{k=0}^{\infty}\left|f\left(\left(s q^{k}\right)+\omega[k]_{q}\right)\right|$ converges uniformly on I, and consequently $f$ is $q$, $\omega$-integrable over $I$.

Lemma $1.5([1,2])$ If $f, g: I \rightarrow \mathbb{R}$ are continuous at $\omega_{0}$, then

$$
\int_{a}^{b} f(t) D_{q, \omega}(g(t)) d_{q, \omega} t=\left.f(t) g(t)\right|_{a} ^{b}-\int_{a}^{b} D_{q, \omega}(f(t)) g(q t+\omega) d_{q, \omega} t, \quad a, b \in I .
$$

Theorem 1.6 ([1,2]) Assume that $f: I \rightarrow \mathbb{R}$ is continuous at $\omega_{0}$. Define

$$
F(x):=\int_{\omega_{0}}^{x} f(t) d_{q, \omega} t
$$

Then $F$ is continuous at $\omega_{0}$. Furthermore, $D_{q, \omega} F(x)$ exists for every $x \in I$ and $D_{q, \omega} F(x)=f(x)$. Conversely,

$$
\int_{a}^{b} D_{q, \omega} f(t) d_{q, \omega} t=f(b)-f(a), \quad a, b \in I .
$$

\section{Main results}

We define the $q, \omega$-derivative of higher order in the usual way. That is, the $n$th $q, \omega$ derivative, $n \in \mathbb{N}$, of $f: I \rightarrow \mathbb{R}$ is the function $D_{q, \omega}^{n} f: I \rightarrow \mathbb{R}$ given by $D_{q, \omega}^{n} f:=D_{q, \omega}\left(D_{q, \omega}^{n-1} f\right)$, provided $D_{q, \omega}^{n-1} f$ is $q, \omega$-differentiable on $I$ and $D_{q, \omega}^{0} f=f$. We consider the following linear spaces:

$$
\begin{aligned}
C^{n} & =C^{n}(I, \mathbb{R}) \\
& :=\left\{f: I \rightarrow \mathbb{R} \mid f \text { is differentiable } n \text {-times and } f^{(i)} \text { are continuous, } i=1,2, \ldots, n\right\}, \\
C_{q, \omega}^{n} & =C_{q, \omega}^{n}(I, \mathbb{R}) \\
& :=\left\{f: I \rightarrow \mathbb{R} \mid f \text { is } q, \omega \text {-differentiable } n \text {-times and } D_{q, \omega}^{n} f \text { is continuous at } \omega_{0}\right\},
\end{aligned}
$$

and

$$
\begin{aligned}
C_{q, \omega}^{\infty} & =C_{q, \omega}^{\infty}(I, \mathbb{R}) \\
& :=\left\{f: I \rightarrow \mathbb{R} \mid f \text { is } q, \omega \text {-differentiable infinitely many times at } \omega_{0}\right\} .
\end{aligned}
$$

Our target is to obtain Taylor expansion of a function $f$ defined on an interval $I$ that contains $\omega_{0}$ associated with Hahn difference operator. We need the following lemmas in proving our main results. 
Lemma 2.1 Let $f$ be a function defined on I. Then, for $x \neq \omega_{0}$, the $n$th $q, \omega$ derivative $\left(D_{q, \omega}^{n} f\right)(x)$ can be expressed as

$$
\left(D_{q, \omega}^{n} f\right)(x)=(x(q-1)+\omega)^{-n} q^{-\frac{n(n-1)}{2}} \sum_{k=0}^{n}\left(\begin{array}{l}
n \\
k
\end{array}\right)_{q}(-1)^{k} q^{\frac{k(k-1)}{2}} f\left(x q^{n-k}+\omega[n-k]_{q}\right) .
$$

Proof For $n=1$, the formula above yields (1.1). Assume that formula (2.1) is true for $n=m$. By relations (1.5), (1.8), and (1.10), we have

$$
\begin{aligned}
\left(D_{q, \omega}^{m+1} f\right)(x)= & D_{q, \omega}\left[(x(q-1)+\omega)^{-m} q^{-\frac{m(m-1)}{2}} \sum_{k=0}^{m}\left(\begin{array}{c}
m \\
k
\end{array}\right)_{q}(-1)^{k} q^{\frac{k(k-1)}{2}}\right. \\
& \left.\times f\left(x q^{m-k}+\omega[m-k]_{q}\right)\right] \\
= & -(q-1) \sum_{j=0}^{m-1}((q x+\omega)(q-1)+\omega)^{-m+j}(x(q-1)+\omega)^{-j-1} \\
& \times q^{-\frac{m(m-1)}{2}} \sum_{k=0}^{m}\left(\begin{array}{c}
m \\
k
\end{array}\right)_{q}(-1)^{k} q^{\frac{k(k-1)}{2}} f\left(x q^{m-k}+\omega[m-k]_{q}\right) \\
& +((q x+\omega)(q-1)+\omega)^{-m} q^{-\frac{m(m-1)}{2}} \sum_{k=0}^{m}\left(\begin{array}{c}
m \\
k
\end{array}\right)_{q}(-1)^{k} q^{\frac{k(k-1)}{2}} \\
& \times D_{q, \omega} f\left(x q^{m-k}+\omega[m-k]_{q}\right) \\
= & q^{-} \frac{m(m-1)}{2} q^{-m}\left[-(q-1) \sum_{j=0}^{m-1} q^{j}(x(q-1)+\omega)^{-m-1}\right. \\
& \times \sum_{k=0}^{m}\left(\begin{array}{l}
m \\
k
\end{array}\right)(-1)^{k} q^{\frac{k(k-1)}{2}} f\left(x q^{m-k}+\omega[m-k]_{q}\right) \\
& +(x(q-1)+\omega)^{-m-1} \sum_{k=0}^{m}\left(\begin{array}{l}
m \\
k
\end{array}\right)(-1)_{q}^{k} q^{\frac{k(k-1)}{2}} \\
& \left.\left(f\left(x q^{m-k+1}+\omega[m-k+1]_{q}\right)-f\left(x q^{m-k}+\omega[m-k]_{q}\right)\right)\right] .
\end{aligned}
$$

This implies that

$$
\begin{aligned}
\left(D_{q, \omega}^{m+1} f\right)(x)= & q^{-\frac{m(m-1)}{2}} q^{-m}(x(q-1)+\omega)^{-m-1}\left[-(q-1) \sum_{j=0}^{m-1} q^{j}\right. \\
& \times \sum_{k=0}^{m}\left(\begin{array}{c}
m \\
k
\end{array}\right)_{q}(-1)^{k} q^{\frac{k(k-1)}{2}} f\left(x q^{m-k}+\omega[m-k]_{q}\right) \\
& +\sum_{k=0}^{m}\left(\begin{array}{c}
m \\
k
\end{array}\right)_{q}(-1)^{k} q^{\frac{k(k-1)}{2}}\left(f\left(x q^{m-k+1}+\omega[m-k+1]_{q}\right)\right. \\
& \left.\left.-f\left(x q^{m-k}+\omega[m-k]_{q}\right)\right)\right]
\end{aligned}
$$


Oraby and Hamza Journal of Inequalities and Applications

(2020) 2020:124

Page 6 of 19

$$
\begin{aligned}
& =q^{-\frac{m(m+1)}{2}}(x(q-1)+\omega)^{-m-1}\left[-(q-1) \frac{q^{m}-1}{q-1} \sum_{k=0}^{m}\left(\begin{array}{l}
m \\
k
\end{array}\right)_{q}\right. \\
& \times(-1)^{k} q^{\frac{k(k-1)}{2}} f\left(x q^{m-k}+\omega[m-k]_{q}\right)+\sum_{k=0}^{m}\left(\begin{array}{l}
m \\
k
\end{array}\right)_{q}(-1)^{k} \\
& \left.\times q^{\frac{k(k-1)}{2}}\left(f\left(x q^{m-k+1}+\omega[m-k+1]_{q}\right)-f\left(x q^{m-k}+\omega[m-k]_{q}\right)\right)\right] \\
& =q^{-\frac{m(m+1)}{2}}(x(q-1)+\omega)^{-m-1}\left[-q^{m} \sum_{k=0}^{m}\left(\begin{array}{l}
m \\
k
\end{array}\right)_{q}(-1)^{k} q^{\frac{k(k-1)}{2}}\right. \\
& \times f\left(x q^{m-k}+\omega[m-k]_{q}\right)+\sum_{k=0}^{m}\left(\begin{array}{c}
m \\
k
\end{array}\right)_{q}(-1)^{k} q^{\frac{k(k-1)}{2}} \\
& \left.\times f\left(x q^{m-k+1}+\omega[m-k+1]_{q}\right)\right] \\
& =q^{-\frac{m(m+1)}{2}}(x(q-1)+\omega)^{-m-1}\left[-q^{m} \sum_{k=1}^{m+1}\left(\begin{array}{c}
m \\
k-1
\end{array}\right)_{q}(-1)^{k-1}\right. \\
& \times q^{\frac{(k-1)(k-2)}{2}} f\left(x q^{m-k+1}+\omega[m-k+1]_{q}\right) \\
& \left.+\sum_{k=0}^{m}\left(\begin{array}{l}
m \\
k
\end{array}\right)_{q}(-1)^{k} q^{\frac{k(k-1)}{2}} f\left(x q^{m-k+1}+\omega[m-k+1]_{q}\right)\right] \\
& =q^{-\frac{m(m+1)}{2}}(x(q-1)+\omega)^{-m-1}\left[\sum_{k=1}^{m+1}\left(\begin{array}{c}
m \\
k-1
\end{array}\right)_{q} q^{m-k+1}(-1)^{k}\right. \\
& \times q^{\frac{k(k-1)}{2}} f\left(x q^{m-k+1}+\omega[m-k+1]_{q}\right) \\
& \left.+\sum_{k=0}^{m}\left(\begin{array}{l}
m \\
k
\end{array}\right)_{q}(-1)^{k} q^{\frac{k(k-1)}{2}} f\left(x q^{m-k+1}+\omega[m-k+1]_{q}\right)\right] \\
& =q^{-\frac{m(m+1)}{2}}(x(q-1)+\omega)^{-m-1}\left[(-1)^{m+1} q^{\frac{m(m+1)}{2}} f(x)\right. \\
& +\sum_{k=1}^{m}\left(\left(\begin{array}{c}
m \\
k-1
\end{array}\right)_{q} q^{m-k+1}+\left(\begin{array}{c}
m \\
k
\end{array}\right)_{q}\right) \\
& \times(-1)^{k} q^{\frac{k(k-1)}{2}} f\left(x q^{m-k+1}+\omega[m-k+1]_{q}\right) \\
& \left.+f\left(x q^{m+1}+\omega[m+1]_{q}\right)\right] \text {. }
\end{aligned}
$$

That is,

$$
\begin{aligned}
\left(D_{q, \omega}^{m+1} f\right)(x)= & q^{-\frac{m(m+1)}{2}}(x(q-1)+\omega)^{-m-1}\left[(-1)^{m+1} q^{\frac{m(m+1)}{2}} f(x)\right. \\
& +\sum_{k=1}^{m}\left(\begin{array}{c}
m+1 \\
k
\end{array}\right)_{q}(-1)^{k} q^{\frac{k(k-1)}{2}} f\left(x q^{m-k+1}+\omega[m-k+1]_{q}\right)
\end{aligned}
$$




$$
\begin{aligned}
& \left.+f\left(x q^{m+1}+\omega[m+1]_{q}\right)\right] \\
= & q^{-\frac{m(m+1)}{2}}(x(q-1)+\omega)^{-m-1} \sum_{k=0}^{m+1}\left[\left(\begin{array}{c}
m+1 \\
k
\end{array}\right)_{q}(-1)^{k} q^{\frac{k(k-1)}{2}}\right. \\
& \left.\times f\left(x q^{m-k+1}+\omega[m-k+1]_{q}\right)\right] .
\end{aligned}
$$

Therefore relation (2.1) is true at $n=m+1$ and by induction it is true for every $n \in \mathbb{N}$.

In the following result, a formula of the $n$th derivative of a power series of center zero is given.

Lemma 2.2 Assume that a function $f$ has the power series expansion $f(x)=\sum_{k=0}^{\infty} a_{k} x^{k}$, $x \in I$. Then

$$
\begin{aligned}
\left(D_{q, \omega}^{n} f\right)(x)= & (1-q)^{-n} \sum_{k=0}^{\infty} \frac{a_{n+k}}{(1-q)^{k}} \sum_{m=0}^{k}(-1)^{m}\left(\begin{array}{c}
n+k \\
n+m
\end{array}\right) \\
& \times(x(q-1)+\omega)^{m}(\omega)^{k-m}\left(q^{m+1} ; q\right)_{n}, \quad x \neq \omega_{0}, n \in \mathbb{N}_{0} .
\end{aligned}
$$

Proof It is clear that Eq. (2.2) is true for $n=0$. From Eq. (2.1) and relation (1.9), we have, for $n \in \mathbb{N}$,

$$
\begin{aligned}
\left(D_{q, \omega}^{n} f\right)(x)= & (x(q-1)+\omega)^{-n} q^{-\frac{n(n-1)}{2}} \sum_{k=0}^{n}\left(\begin{array}{l}
n \\
k
\end{array}\right)_{q}(-1)^{k} q^{\frac{k(k-1)}{2}} \\
& \times \sum_{j=0}^{\infty} a_{j}\left(x q^{n-k}+\omega[n-k]_{q}\right)^{j} \\
= & (x(q-1)+\omega)^{-n} q^{-\frac{n(n-1)}{2}} \sum_{j=0}^{\infty} \frac{a_{j}}{(1-q)^{j}} \sum_{r=0}^{j}(-1)^{r}\left(\begin{array}{l}
j \\
r
\end{array}\right) q^{n r} \\
& \times(x(q-1)+\omega)^{r}(\omega)^{j-r} \sum_{k=0}^{n}\left(\begin{array}{l}
n \\
k
\end{array}\right)_{q}(-1)^{k} q^{\frac{k(k-1)}{2}} q^{-k r} \\
= & (x(q-1)+\omega)^{-n} q^{-\frac{n(n-1)}{2}} \sum_{j=0}^{\infty} \frac{a_{j}}{(1-q)^{j}} \sum_{r=0}^{j}(-1)^{r}\left(\begin{array}{l}
j \\
r
\end{array}\right) q^{n r} \\
& \times(x(q-1)+\omega)^{r}(\omega)^{j-r}\left(q^{-r} ; q\right)_{n} .
\end{aligned}
$$

Then

$$
\begin{aligned}
\left(D_{q, \omega}^{n} f\right)(x)= & (-1)^{n}(x(q-1)+\omega)^{-n} q^{-\frac{n(n-1)}{2}} \sum_{j=n}^{\infty} \frac{a_{j}}{(1-q)^{j}} \sum_{r=n}^{j}(-1)^{r} q^{n r}\left(\begin{array}{l}
j \\
r
\end{array}\right) \\
& \times(x(q-1)+\omega)^{r}(\omega)^{j-r} q^{-r n+\frac{n(n-1)}{2}}\left(q^{r-n+1} ; q\right)_{n} \\
= & (-1)^{n}(x(q-1)+\omega)^{-n} \sum_{j=n}^{\infty} \frac{a_{j}}{(1-q)^{j}} \sum_{r=n}^{j}(-1)^{r}\left(\begin{array}{l}
j \\
r
\end{array}\right)
\end{aligned}
$$




$$
\begin{aligned}
& \times(x(q-1)+\omega)^{r}(\omega)^{j-r}\left(q^{r-n+1} ; q\right)_{n} \\
= & (-1)^{n}(x(q-1)+\omega)^{-n} \sum_{k=0}^{\infty} \frac{a_{n+k}}{(1-q)^{n+k}} \sum_{r=n}^{n+k}(-1)^{r}\left(\begin{array}{c}
n+k \\
r
\end{array}\right) \\
& \times(x(q-1)+\omega)^{r}(\omega)^{n+k-r}\left(q^{r-n+1} ; q\right)_{n} \\
= & (-1)^{n}(x(q-1)+\omega)^{-n} \sum_{k=0}^{\infty} \frac{a_{n+k}}{(1-q)^{n+k}} \sum_{m=0}^{k}(-1)^{n+m}\left(\begin{array}{c}
n+k \\
n+m
\end{array}\right) \\
& \times(x(q-1)+\omega)^{n+m}(\omega)^{k-m}\left(q^{m+1} ; q\right)_{n} \\
= & (1-q)^{-n} \sum_{k=0}^{\infty} \frac{a_{n+k}}{(1-q)^{k}} \sum_{m=0}^{k}(-1)^{m}\left(\begin{array}{c}
n+k \\
n+m
\end{array}\right)(x(q-1)+\omega)^{m} \\
& \times(\omega)^{k-m}\left(q^{m+1} ; q\right)_{n} \\
= & (1-q)^{-n} \sum_{k=0}^{\infty} \frac{a_{n+k}}{(1-q)^{k}}\left[(-1)^{k}(x(q-1)+\omega)^{k}\left(q^{k+1} ; q\right)_{n}\right. \\
& \left.+\sum_{m=0}^{k-1}(-1)^{m}\left(\begin{array}{c}
n+k \\
n+m
\end{array}\right)(x(q-1)+\omega)^{m}(\omega)^{k-m}\left(q^{m+1} ; q\right)_{n}\right] .
\end{aligned}
$$

The following result includes a useful formula for the $n$th derivative of a power series of center $\omega_{0}$.

Lemma 2.3 Assume that a function $f$ has the power series expansion $f(x)=\sum_{k=0}^{\infty} a_{k}(x-$ $\left.\omega_{0}\right)^{k}, x \in I$. Then

$$
D_{q, \omega}^{n} f(x)=(x(1-q)-\omega)^{-n} \sum_{k=0}^{\infty} a_{n+k}\left(x-\omega_{0}\right)^{n+k}\left(q^{k+1} ; q\right)_{n}, \quad x \neq \omega_{0} .
$$

Proof It is clear that Eq. (2.3) is true for $n=0$. From Eq. (2.1) and relation (1.9), we have, for $n \in \mathbb{N}$,

$$
\begin{aligned}
\left(D_{q, \omega}^{n} f\right)(x)= & (x(q-1)+\omega)^{-n} q^{-\frac{n(n-1)}{2}} \sum_{k=0}^{n}\left[\left(\begin{array}{l}
n \\
k
\end{array}\right)_{q}(-1)^{k} q^{\frac{k(k-1)}{2}}\right. \\
& \left.\times \sum_{j=0}^{\infty} a_{j}\left(x q^{n-k}+\omega[n-k]_{q}-\omega_{0}\right)^{j}\right]
\end{aligned}
$$

From this it follows that

$$
\begin{aligned}
\left(D_{q, \omega}^{n} f\right)(x)= & (x(q-1)+\omega)^{-n} q^{-\frac{n(n-1)}{2}} \sum_{k=0}^{n}\left[\left(\begin{array}{l}
n \\
k
\end{array}\right)_{q}(-1)^{k} q^{\frac{k(k-1)}{2}}\right. \\
& \left.\times \sum_{j=0}^{\infty} a_{j} q^{n j-k j}\left(x-\omega_{0}\right)^{j}\right]
\end{aligned}
$$




$$
\begin{aligned}
= & (x(q-1)+\omega)^{-n} q^{-\frac{n(n-1)}{2}} \sum_{j=0}^{\infty}\left[a_{j} q^{n j}\left(x-\omega_{0}\right)^{j}\right. \\
& \left.\times \sum_{k=0}^{n}\left(\begin{array}{l}
n \\
k
\end{array}\right)_{q}(-1)^{k} q^{\frac{k(k-1)}{2}} q^{-k j}\right] \\
= & (x(q-1)+\omega)^{-n} q^{-\frac{n(n-1)}{2}} \sum_{j=0}^{\infty} a_{j} q^{n j}\left(x-\omega_{0}\right)^{j}\left(q^{-j} ; q\right)_{n} \\
= & (x(q-1)+\omega)^{-n} q^{-\frac{n(n-1)}{2}} \sum_{j=n}^{\infty}\left[a_{j} q^{n j}\left(x-\omega_{0}\right)^{j}(-1)^{n} q^{-n j+\frac{n(n-1)}{2}}\right. \\
& \left.\times\left(q^{j-n+1} ; q\right)_{n}\right]^{-n} \sum_{k=0}^{\infty} a_{n+k}\left(x-\omega_{0}\right)^{n+k}\left(q^{k+1} ; q\right)_{n} . \\
= & (x(1-q)-\omega)^{-}
\end{aligned}
$$

One of the important questions: Is there a relation between the $n$th $q, \omega$ derivative and the usual $n$th derivative? The answer is in the following lemma.

Lemma 2.4 Iff $\in C^{n+1}$, then

(i) $D_{q, \omega}^{m} f$ exists on $I$ and is continuous at $\omega_{0}$ for all $m=1,2, \ldots, n+1$;

(ii) for $1 \leq m \leq n+1$,

$$
D_{q, \omega}^{m} f\left(\omega_{0}\right)=\frac{[m]_{q} !}{m !} f^{(m)}\left(\omega_{0}\right)
$$

where $f^{(m)}$ is the usual mth derivative off.

Proof The proof is by induction. The $q, \omega$ derivative $D_{q, \omega} f$ exists and $D_{q, \omega} f\left(\omega_{0}\right)=f^{\prime}\left(\omega_{0}\right)$. Also $D_{q, \omega} f$ is continuous at $\omega_{0}$. Indeed,

$$
\lim _{x \rightarrow \omega_{0}} D_{q, \omega} f(x)=\lim _{t \rightarrow \omega_{0}} \frac{f(q x+\omega)-f(x)}{x(q-1)+\omega}=f^{\prime}\left(\omega_{0}\right)=D_{q, \omega} f\left(\omega_{0}\right) .
$$

Now, we assume that (i) and (ii) hold for all $m=1,2, \ldots, l$, where $l \leq n$ and we want to prove that they are true at $m=l+1$. By Lemma 2.1, we conclude that

$$
\begin{aligned}
\lim _{x \rightarrow \omega_{0}} D_{q, \omega}^{l+1} f(x)= & \lim _{x \rightarrow \omega_{0}} \frac{1}{(x(q-1)+\omega)^{l+1} q^{\frac{l(l+1)}{2}}}\left[\sum_{k=0}^{l+1}\left(\begin{array}{c}
l+1 \\
k
\end{array}\right)_{q}(-1)^{k} q^{\frac{k(k-1)}{2}}\right. \\
& \left.\times f\left(x q^{l-k+1}+\omega[l-k+1]_{q}\right)\right] \\
= & \lim _{x \rightarrow \omega_{0}} \sum_{k=0}^{l+1}\left[\frac{\left(\begin{array}{c}
l+1 \\
k
\end{array}\right)_{q}(-1)^{k} q^{\frac{k(k-1)}{2}} q^{(l+1)(l-k+1)}}{(q-1)^{l+1}\left(x q^{l-k+1}+\omega[l-k+1]_{q}-\omega_{0}\right)^{l+1} q^{\frac{l(l+1)}{2}}}\right. \\
& \left.\times f\left(x q^{l-k+1}+\omega[l-k+1]_{q}\right)\right] .
\end{aligned}
$$


Applying L'Hopital rule $l+1$ times and using relations (1.12), (1.13), and (1.14), we get

$$
\begin{aligned}
\lim _{x \rightarrow \omega_{0}} D_{q, \omega}^{l+1} f(x)= & \lim _{x \rightarrow \omega_{0}} \frac{1}{(q-1)^{l+1}(l+1) ! q^{\frac{l(l+1)}{2}}} \sum_{k=0}^{l+1}\left[\left(\begin{array}{c}
l+1 \\
k
\end{array}\right)_{q}(-1)^{k} q^{\frac{k(k-1)}{2}}\right. \\
& \left.\times q^{(l+1)(l-k+1)} f^{(l+1)}\left(x q^{l-k+1}+\omega[l-k+1]_{q}\right)\right] \\
= & \frac{\sum_{k=0}^{l+1}\left(\begin{array}{c}
l+1 \\
k
\end{array}\right)_{q}(-1)^{k} q^{\frac{k(k-1)}{2}}\left(q^{l+1}\right)^{l-k+1} f^{(l+1)}\left(\omega_{0}\right)}{(q-1)^{l+1}(l+1) ! q^{\frac{l(l+1)}{2}}} \\
= & \frac{\left[q^{l+1}-1\right]_{l+1} f^{(l+1)}\left(\omega_{0}\right)}{(q-1)^{l+1}(l+1) ! q^{\frac{l l+1)}{2}}} \\
= & \frac{\left(q^{l+1}-1\right)\left(q^{l+1}-q\right)\left(q^{l+1}-q^{2}\right) \cdots\left(q^{l+1}-q^{l}\right) f^{(l+1)}\left(\omega_{0}\right)}{(q-1)^{l+1}(l+1) ! q^{0+1+2+\cdots+(l-1)+l}} \\
= & \frac{\left(q^{l+1}-1\right)\left(q^{l}-1\right)\left(q^{l-1}-1\right) \cdots(q-1) f^{(l+1)}\left(\omega_{0}\right)}{(q-1)^{l+1}(l+1) !} \\
= & \frac{[1]_{q}[2]_{q} \cdots[l]_{q}[l+1]_{q} f^{(l+1)}\left(\omega_{0}\right)}{(l+1) !} \\
= & \frac{[l+1]_{q} !}{(l+1) !} f^{(l+1)}\left(\omega_{0}\right) .
\end{aligned}
$$

On the other hand, we conclude that

$$
\begin{aligned}
D_{q, \omega}^{l+1} f\left(\omega_{0}\right)= & \lim _{x \rightarrow \omega_{0}} \frac{D_{q, \omega}^{l} f(x)-D_{q, \omega}^{l} f\left(\omega_{0}\right)}{x-\omega_{0}} \\
= & \lim _{x \rightarrow \omega_{0}} \frac{d}{d x}\left[\frac{\sum_{k=0}^{l}\left(\begin{array}{l}
l \\
k
\end{array}\right)_{q}(-1)^{k} q^{\frac{k(k-1)}{2}} f\left(x q^{l-k}+\omega[l-k]_{q}\right)}{(x(q-1)+\omega)^{l} q^{\frac{l(l-1)}{2}}}\right] \\
= & \lim _{x \rightarrow \omega_{0}} \frac{1}{(x(q-1)+\omega)^{l+1} q^{\frac{l(l-1)}{2}}} \sum_{k=0}^{l}\left(\begin{array}{l}
l \\
k
\end{array}\right)_{q}(-1)^{k} q^{\frac{k(k-1)}{2}} \\
& \times\left[(x(q-1)+\omega) q^{l-k} f^{\prime}\left(x q^{l-k}+\omega[l-k]_{q}\right)-l(q-1) f\left(x q^{l-k}+\omega[l-k]_{q}\right)\right] .
\end{aligned}
$$

Again, applying L'Hopital rule $l+1$ times and using relations (1.12), (1.13), and (1.14), we get

$$
\begin{aligned}
D_{q, \omega}^{l+1} f\left(\omega_{0}\right)= & \lim _{x \rightarrow \omega_{0}} \frac{1}{(q-1)^{l+1}(l+1) ! q^{\frac{l(l-1)}{2}}} \sum_{k=0}^{l}\left[\left(\begin{array}{l}
l \\
k
\end{array}\right)_{q}(-1)^{k} q^{\frac{k(k-1)}{2}} q^{(l+1)(l-k)}\right. \\
& \left.\times(q-1) f^{(l+1)}\left(x q^{l-k}+\omega[l-k]_{q}\right)\right] \\
= & \frac{\left[q^{l+1}-1\right]_{l}(q-1) f^{(l+1)}\left(\omega_{0}\right)}{(q-1)^{l+1}(l+1) ! q^{\frac{l(l-1)}{2}}} \\
= & \frac{[l+1]_{q} !}{(l+1) !} f^{(l+1)}\left(\omega_{0}\right) .
\end{aligned}
$$


Therefore,

$$
\lim _{x \rightarrow \omega_{0}} D_{q, \omega}^{l+1} f(x)=D_{q, \omega}^{l+1} f\left(\omega_{0}\right)=\frac{[l+1]_{q} !}{(l+1) !} f^{(l+1)}\left(\omega_{0}\right)
$$

Corollary 2.5 Assume that $f$ has the power series expansion

$$
f(x)=\sum_{n=0}^{\infty} a_{n}\left(x-\omega_{0}\right)^{n}, \quad x \in I .
$$

Then

$$
a_{n}=\frac{D_{q, \omega}^{n} f\left(\omega_{0}\right)}{[n]_{q} !}, \quad n \in \mathbb{N} .
$$

Proof By Lemma 2.4, we have

$$
a_{n}=\frac{f^{(n)}\left(\omega_{0}\right)}{n !}=\frac{D_{q, \omega}^{n} f\left(\omega_{0}\right)}{[n]_{q} !} .
$$

Now we define the two variable polynomials $H_{n}(x, t), x, t \in I$, to be

$$
H_{0}(x, t):=1, \quad H_{n}(x, t):=\prod_{j=0}^{n-1}\left(x-h^{j}(t)\right),
$$

where $h^{j}(t)=t q^{j}+\omega[j]_{q}, t \in I$ is the $j$ th order iteration of $h(t)=q t+\omega$, which uniformly converges to $\omega_{0}$ on $I$.

Lemma 2.6 For $n \in \mathbb{N}$ and $x, t \in I$, we have

$$
\begin{aligned}
& { }_{t} D_{q, \omega} H_{n}(x, t)=-[n]_{q} H_{n-1}(x, h(t)), \\
& { }_{x} D_{q, \omega} H_{n}(x, t)=[n]_{q} H_{n-1}(x, t),
\end{aligned}
$$

where ${ }_{t} D_{q, \omega}$ is the $q, \omega$-derivative with respect to $t$,

$$
I_{q, \omega}^{n}(1)=\frac{H_{n}(x, a)}{\Gamma_{q}(n+1)}
$$

where $I_{q, \omega}^{n}$ is the $q, \omega$-integral

$$
I_{q, \omega}^{n} f(x):=\int_{a}^{x} \int_{a}^{x_{n-1}} \int_{a}^{x_{n-2}} \cdots \int_{a}^{x_{1}} f(s) d_{q, \omega} s d_{q, \omega} x_{1} \cdots d_{q, \omega} x_{n-2} d_{q, \omega} x_{n-1} .
$$

Now, we establish Taylor's theorem based on Hahn difference operator.

Theorem 2.7 Let $f$ be a function defined on I. If $\in C_{q, \omega}^{n}$ for some $n \in \mathbb{N}$, then for $x, a \in I$,

$$
f(x)=\sum_{k=0}^{n-1} \frac{D_{q, \omega}^{k} f(a)}{[k]_{q} !} H_{k}(x, a)+R_{n}(x, a),
$$


where

$$
R_{n}(x, a)=\int_{a}^{x} \frac{D_{q, \omega}^{n} f(t)}{[n-1]_{q} !} H_{n-1}(x, h(t)) d_{q, \omega} t
$$

Proof We prove relation (2.9) by induction. The right-hand side (R.H.S) of (2.9) at $n=1$ is

$$
\begin{aligned}
\text { R.H.S } & =f(a) H_{0}(x, a)+R_{1}(x, a) \\
& =f(a)+\int_{a}^{x} D_{q, \omega} f(t) d_{q, \omega} t=f(x) .
\end{aligned}
$$

Assume that relation (2.9) is true for $n=m$, that is,

$$
f(x)=\sum_{k=0}^{m-1} \frac{D_{q, \omega}^{k} f(a)}{[k]_{q} !} H_{k}(x, a)+R_{m}(x, a),
$$

where $R_{m}(x, a)=\int_{a}^{x} \frac{D_{q, \omega}^{m} f(t)}{[m-1]_{q} !} H_{m-1}(x, h(t)) d_{q, \omega} t$. We integrate by parts in the remainder term $R_{m}(x, a)$. We obtain

$$
\begin{aligned}
R_{m}(x, a) & =\int_{a}^{x} \frac{D_{q, \omega}^{m} f(t)}{[m-1]_{q} !} H_{m-1}(x, h(t)) d_{q, \omega} t \\
& =-\int_{a}^{x} \frac{D_{q, \omega}^{m} f(t)}{[m-1]_{q} !} \frac{{ }_{q, \omega} D_{m}(x, t)}{[m]_{q}} d_{q, \omega} t \\
& =-\left.\frac{D_{q, \omega}^{m} f(t)}{[m]_{q} !} H_{m}(x, t)\right|_{a} ^{x}+\int_{a}^{x} \frac{D_{q, \omega}^{m+1} f(t)}{[m]_{q} !} H_{m}(x, h(t)) d_{q, \omega} t \\
& =D_{q, \omega}^{m} f(a) \frac{H_{m}(x, a)}{[m]_{q} !}+R_{m+1}(x, a) .
\end{aligned}
$$

Then

$$
f(x)=\sum_{k=0}^{m} \frac{D_{q, \omega}^{k} f(a)}{[k]_{q} !} H_{k}(x, a)+R_{m+1}(x, a)
$$

Therefore, relation (2.9) is true for $n=m+1$, then it is true for every $n \in \mathbb{N}$.

As a direct consequence of the previous theorem, we deduce the following theorem.

Theorem 2.8 Let $f \in C_{q, \omega}^{\infty}$. If for $x, a \in I, \lim _{n \rightarrow \infty} R_{n}(x, a)=0$, then $f(x)$ has the following expansion:

$$
f(x)=\sum_{k=0}^{\infty} \frac{D_{q, \omega}^{k} f(a)}{[k]_{q} !} H_{k}(x, a)
$$

Furthermore, if $\lim _{n \rightarrow \infty} R_{n}(x, a)=0$ uniformly with respect to $x$ in some subinterval of $I$, then the series given by (2.11) is uniformly convergent in this subinterval. 
Corollary 2.9 Let $f \in C_{q, \omega}^{\infty}$. If for $x \in I, \lim _{n \rightarrow \infty} R_{n}\left(x, \omega_{0}\right)=0$, then $f(x)$ has the following expansion:

$$
f(x)=\sum_{k=0}^{\infty} \frac{D_{q, \omega}^{k} f\left(\omega_{0}\right)}{[k]_{q} !}\left(x-\omega_{0}\right)^{k}
$$

Theorem 2.10 Let $f \in C_{q, \omega}^{\infty}$. Assume that there is a nonnegative sequence $\left\{M_{n}\right\}$ such that

(i) $\left|D_{q, \omega}^{n} f\left(h^{m}(y)\right)\right| \leq C M_{n}, n, m \in \mathbb{N}_{0}, y \in I$, for some $C>0$;

(ii) $\lim _{n \rightarrow \infty} \frac{M_{n+1}}{M_{n}}=M$ exists.

Then $f$ has the $q, \omega$-Taylor expansion

$$
f(x)=\sum_{k=0}^{\infty} \frac{D_{q, \omega}^{k} f(a)}{[k]_{q} !} H_{k}(x, a)
$$

for every $x \in\left(\omega_{0}-\frac{1}{M(1-q)}, \omega_{0}+\frac{1}{M(1-q)}\right)$ when $M>0$ (respectively $x \in I$ when $\left.M=0\right)$.

Proof We can write $R_{n}(x, a)$ as follows:

$$
R_{n}(x, a)=R_{1, n}\left(x, \omega_{0}\right)-R_{2, n}\left(x ; a, \omega_{0}\right),
$$

where

$$
R_{1, n}\left(x, \omega_{0}\right):=\frac{1}{\Gamma_{q}(n)} \int_{\omega_{0}}^{x} H_{n-1}(x, h(t)) D_{q, \omega}^{n} f(t) d_{q, \omega} t
$$

and

$$
R_{2, n}\left(x ; a, \omega_{0}\right):=\frac{1}{\Gamma_{q}(n)} \int_{\omega_{0}}^{a} H_{n-1}(x, h(t)) D_{q, \omega}^{n} f(t) d_{q, \omega} t .
$$

From (1.16), we have

$$
\begin{aligned}
R_{1, n}\left(x, \omega_{0}\right)= & (x(1-q)-\omega) \sum_{m=0}^{\infty} q^{m} \frac{1}{\Gamma_{q}(n)} H_{n-1}\left(x, h^{m+1}(x)\right) D_{q, \omega}^{n} f\left(h^{m}(x)\right) \\
= & \frac{1}{\Gamma_{q}(n)}(x(1-q)-\omega) \sum_{m=0}^{\infty}\left[q^{m} \prod_{r=0}^{n-2}\left(x-\left[x q^{m+1+r}+[m+1+r]_{q} \omega\right]\right)\right. \\
& \left.\times D_{q, \omega}^{n} f\left(h^{m}(x)\right)\right] \\
= & \frac{(1-q)\left(x-\omega_{0}\right)}{[n-1]_{q} !} \sum_{m=0}^{\infty} q^{m}\left(x-\omega_{0}\right)^{n-1} \prod_{r=0}^{n-2}\left(1-q^{m+r+1}\right) D_{q, \omega}^{n} f\left(h^{m}(x)\right) \\
= & \frac{(1-q)\left(x-\omega_{0}\right)^{n}}{[n-1]_{q} !} \sum_{m=0}^{\infty} q^{m}\left(q^{m+1} ; q\right)_{n-1} D_{q, \omega}^{n} f\left(h^{m}(x)\right) \\
= & \frac{(1-q)^{n}\left(x-\omega_{0}\right)^{n}}{(q ; q)_{n-1}} \sum_{m=0}^{\infty} q^{m}\left(q^{m+1} ; q\right)_{n-1} D_{q, \omega}^{n} f\left(h^{m}(x)\right) .
\end{aligned}
$$


Consequently,

$$
\begin{aligned}
\left|R_{1, n}\left(x, \omega_{0}\right)\right| & \leq \frac{C}{(q ; q)_{\infty}} M_{n}\left[(1-q)\left|x-\omega_{0}\right|\right]^{n} \sum_{m=0}^{\infty} q^{m} \\
& \leq \frac{C M_{n}\left[(1-q)\left|x-\omega_{0}\right|\right]^{n}}{(q ; q)_{\infty}(1-q)} .
\end{aligned}
$$

Then $\lim _{n \rightarrow \infty} R_{1, n}\left(x, \omega_{0}\right)=0, x \in\left(\omega_{0}-\frac{1}{M(1-q)}, \omega_{0}+\frac{1}{M(1-q)}\right)$, when $M>0$ (respectively $x \in I$, when $M=0)$. On the other hand, for $a \in I$, we have

$$
R_{2, n}\left(x ; a, \omega_{0}\right)=\frac{(a(1-q)-\omega)}{\Gamma_{q}(n)} \sum_{m=0}^{\infty} q^{m} H_{n-1}\left(x, h^{m+1}(a)\right) D_{q, \omega}^{n} f\left(h^{m}(a)\right) .
$$

Simple calculations show that

$$
\begin{aligned}
\left|H_{n-1}\left(x, h^{m+1}(a)\right)\right| & =\left|\prod_{r=0}^{n-2}\left(x-h^{m+r+1}(a)\right)\right| \\
& \leq \prod_{r=0}^{n-2}\left[\left|x-\omega_{0}\right|+q^{m+r+1}\left|a-\omega_{0}\right|\right] \\
& \leq\left|x-\omega_{0}\right|^{n-1} e^{\sum_{r=0}^{\infty} q^{m+r+1} \mid \frac{\left|a-\omega_{0}\right|}{\left|x-\omega_{0}\right|}} \\
& \leq\left|x-\omega_{0}\right|^{n-1} e^{\frac{\left|a-\omega_{0}\right|}{(1-q)\left|x-\omega_{0}\right|}} .
\end{aligned}
$$

Consequently,

$$
\begin{aligned}
\left|R_{2, n}\left(x, a, \omega_{0}\right)\right| & \leq \frac{\left|x-\omega_{0}\right|^{n-1}(1-q)\left|a-\omega_{0}\right|}{[n-1]_{q} !} C M_{n} e^{\frac{\left|a-\omega_{0}\right|}{(1-q)\left|x-\omega_{0}\right|}} \sum_{m=0}^{\infty} q^{m} \\
& \leq \frac{C\left|a-\omega_{0}\right| M_{n}\left[(1-q)\left|x-\omega_{0}\right|\right]^{n-1}}{(q, q)_{\infty}} e^{\frac{\left|a-\omega_{0}\right|}{(1-q)\left|x-\omega_{0}\right|}} .
\end{aligned}
$$

This implies that $\lim _{n \rightarrow \infty} R_{2, n}\left(x ; a, \omega_{0}\right)=0, x \in\left(\omega_{0}-\frac{1}{M(1-q)}, \omega_{0}+\frac{1}{M(1-q)}\right)$, when $M>0$ (respectively $x \in I$, when $M=0$ ). Therefore

$$
\lim _{n \rightarrow \infty} R_{n}(x, a)=\lim _{n \rightarrow \infty}\left[R_{1, n}\left(x, \omega_{0}\right)-R_{2, n}\left(x ; a, \omega_{0}\right)\right]=0,
$$

$x \in\left(\omega_{0}-\frac{1}{M(1-q)}, \omega_{0}+\frac{1}{M(1-q)}\right)$, when $M>0($ respectively $x \in I$, when $M=0)$.

Theorem 2.11 Assume that $f$ has the power series expansion $f(x)=\sum_{n=0}^{\infty} a_{n}\left(x-\omega_{0}\right)^{n}$ with interval of convergence $I_{r}=\left(\omega_{0}-r, \omega_{0}+r\right), r>0$. Then, for any $a \in I_{r}, f$ has the $q, \omega$-Taylor expansion

$$
f(x)=\sum_{k=0}^{\infty} \frac{D_{q, \omega}^{k} f(a)}{[k]_{q} !} H_{k}(x, a),
$$

in any closed subinterval $\overline{I_{\alpha}}, \alpha<r$, where the series is absolutely and uniformly convergent on $\overline{I_{\alpha}}, \alpha<r$. 
Proof For $n, m \in \mathbb{N}$ and by Lemma 2.3, we get

$$
\begin{aligned}
D_{q, \omega}^{n} f\left(h^{m}(y)\right) & =\left(h^{m}(y)(1-q)-\omega\right)^{-n} \sum_{k=0}^{\infty} a_{n+k}\left(h^{m}(y)-\omega_{0}\right)^{n+k}\left(q^{k+1} ; q\right)_{n} \\
& =q^{-m n}(y(1-q)-\omega)^{-n} \sum_{k=0}^{\infty} a_{n+k} q^{m n+m k}\left(y-\omega_{0}\right)^{n+k}\left(q^{k+1} ; q\right)_{n} \\
& =\frac{1}{(1-q)^{n}} \sum_{k=0}^{\infty} a_{k} q^{m k}\left(y-\omega_{0}\right)^{k}\left(q^{k+1} ; q\right)_{n} .
\end{aligned}
$$

Consequently, for $\alpha<r$,

$$
\begin{aligned}
\left|D_{q, \omega}^{n} f\left(h^{m}(y)\right)\right| & \leq \frac{1}{(1-q)^{n}} \sum_{k=0}^{\infty}\left|a_{k}\left(y-\omega_{0}\right)^{k}\right| q^{m k} \\
& \leq \frac{1}{(1-q)^{n}} \sum_{k=0}^{\infty}\left|a_{k} \alpha^{k}\right| q^{m k} \\
& \leq \frac{1}{(1-q)^{n}} C, y \in \overline{I_{\alpha}},
\end{aligned}
$$

where $C=\sum_{k=0}^{\infty}\left|a_{k} \alpha^{k}\right|$. Then, by Theorem 2.10, $f$ has the $q, \omega$-Taylor expansion (2.13)

Now, we establish some properties of the $q, \omega$-exponential functions $e_{q, \omega}(t)$ and $E_{q, \omega}(t)$ for $t \in \mathbb{R},\left|t-\omega_{0}\right|<\frac{1}{1-q}$, where

$$
\begin{aligned}
e_{q, \omega}(t) & =\frac{1}{\prod_{k=0}^{\infty}\left(1-q^{k}(t(1-q)-\omega)\right)} \\
& =\frac{1}{((t(1-q)-\omega) ; q)_{\infty}}
\end{aligned}
$$

and

$$
\begin{aligned}
E_{q, \omega}(t) & =\prod_{k=0}^{\infty}\left(1+q^{k}(t(1-q)-\omega)\right) \\
& =(-(t(1-q)-\omega) ; q)_{\infty}
\end{aligned}
$$

Simple calculations show that the following inequalities are true:

$$
\frac{e^{-\frac{q}{1-q}}}{(1-(t(1-q)-\omega))}<e_{q, \omega}(t)<\frac{e^{A}}{1-(t(1-q)-\omega)}, \quad\left|t-\omega_{0}\right|<\frac{1}{1-q}
$$

and

$$
(1+(t(1-q)-\omega)) e^{-A}<E_{q, \omega}(t)<(1+(t(1-q)-\omega)) e^{\frac{q}{1-q}}, \quad\left|t-\omega_{0}\right|<\frac{1}{1-q}
$$

where $A=\sum_{k=1}^{\infty} \frac{q^{k}}{1-q^{k}}$.

Finally, we can prove the following power series expansions for $e_{q, \omega}$ and $E_{q, \omega}$. 
Example 2.12 The exponential functions $e_{q, \omega}$ and $E_{q, \omega}$ defined in (2.14) and (2.15) have the following power series expansions of center $a \in I$ :

$$
e_{q, \omega}(x)=\sum_{k=0}^{\infty} \frac{e_{q, \omega}(a)}{[k]_{q} !} H_{k}(x, a), \quad\left|x-\omega_{0}\right|<\frac{1}{1-q}
$$

and

$$
E_{q, \omega}(x)=\sum_{k=0}^{\infty} \frac{q^{\frac{k(k-1)}{2}} E_{q, \omega}\left(h^{k}(a)\right)}{[k]_{q} !} H_{k}(x, a), \quad x \in I,
$$

and have the following power series expansions of center $\omega_{0}$ :

$$
e_{q, \omega}(t)=\sum_{k=0}^{\infty} \frac{1}{[k]_{q} !}\left(t-\omega_{0}\right)^{k}
$$

and

$$
E_{q, \omega}(t)=\sum_{k=0}^{\infty} \frac{q^{\frac{k(k-1)}{2}}}{[k]_{q} !}\left(t-\omega_{0}\right)^{k} .
$$

Furthermore, both $e_{q, \omega}$ and $E_{q, \omega}$ are continuous.

Proof For $n \in \mathbb{N}_{0}$, we have

$$
D_{q, \omega}^{n} e_{q, \omega}(t)=e_{q, \omega}(t)
$$

Inequality (2.16) shows that $e_{q, \omega}(t)$ is positive and bounded on every compact subinterval of $\left(\omega_{0}-\frac{1}{1-q}, \omega_{0}+\frac{1}{1-q}\right)$. For fixed $t \in\left(\omega_{0}-\frac{1}{1-q}, \omega_{0}+\frac{1}{1-q}\right)$, there exists $0<\alpha \leq 1$ such that $|t(1-q)-\omega|<\alpha$, which implies that

$$
\left|D_{q, \omega}^{n} e_{q, \omega}(t)\right| \leq \frac{e^{A}}{1-\alpha}, \quad n \in \mathbb{N}_{0}
$$

By Theorem 2.10, the $q, \omega$-Taylor expansion of $e_{q, \omega}(t)$ at $a$ is given by

$$
e_{q, \omega}(t)=\sum_{k=0}^{\infty} \frac{e_{q, \omega}(a)}{[k]_{q} !} H_{k}(t, a)
$$

Since $D_{q, \omega}^{n} e_{q, \omega}\left(\omega_{0}\right)=1$, the $q, \omega$-Taylor expansion of $e_{q, \omega}(t)$ at $\omega_{0}$ is given by

$$
e_{q, \omega}(t)=\sum_{k=0}^{\infty} \frac{1}{[k]_{q} !}\left(t-\omega_{0}\right)^{k}
$$

The series in (2.23) is uniformly convergent on every compact subinterval of $\left(\omega_{0}-\frac{1}{1-q}\right.$, $\left.\omega_{0}+\frac{1}{1-q}\right)$ by Weierstrass M-test, and consequently $e_{q, \omega}(t)$ is continuous. 
Let $t \in \mathbb{R},\left|t-\omega_{0}\right|<\frac{1}{1-q}$. First, we show that

$$
D_{q, \omega}^{n} E_{q, \omega}(t)=q^{\frac{n(n-1)}{2}} E_{q, \omega}\left(h^{n}(t)\right), \quad n \in \mathbb{N}_{0}
$$

by induction. For $n=1$, we have

$$
\begin{aligned}
D_{q, \omega} E_{q, \omega}(t)= & \frac{1}{t(q-1)+\omega}\left[\prod_{k=0}^{\infty}\left(1+q^{k}(q t+\omega)(1-q)-\omega\right)\right) \\
& \left.-\prod_{k=0}^{\infty}\left(1+q^{k}(t(1-q)-\omega)\right)\right] \\
= & \frac{\prod_{k=0}^{\infty}\left(1+q^{k+1}(t(1-q)-\omega)\right)}{t(q-1)+\omega}[1-(1+t(1-q)-\omega)] \\
= & E_{q, \omega}(h(t)) .
\end{aligned}
$$

Assume that formula (2.24) is true for $n=m$. We have

$$
\begin{aligned}
D_{q, \omega}^{m+1} E_{q, \omega}(t)= & D_{q, \omega}\left(D_{q, \omega}^{m} E_{q, \omega}(t)\right) \\
= & q^{\frac{m(m-1)}{2}} D_{q, \omega} E_{q, \omega}\left(h^{m}(t)\right) \\
= & q^{\frac{m(m-1)}{2}} \frac{1}{t(q-1)+\omega}\left[\prod_{k=0}^{\infty}\left(1+q^{k+m+1}(t(1-q)-\omega)\right)\right. \\
& \left.-\prod_{k=0}^{\infty}\left(1+q^{k+m}(t(1-q)-\omega)\right)\right] \\
= & q^{\frac{m(m-1)}{2}} \frac{\prod_{k=0}^{\infty}\left(1+q^{k+m+1}(t(1-q)-\omega)\right)}{t(q-1)+\omega} \\
& \times\left[1-\left(1+q^{m}(t(1-q)-\omega)\right)\right] \\
= & q^{\frac{m(m+1)}{2}} \prod_{k=0}^{\infty}\left(1+q^{k+m+1}(t(1-q)-\omega)\right) \\
= & q^{\frac{m(m+1)}{2}} E_{q, \omega}\left(h^{m+1}(t)\right) .
\end{aligned}
$$

Inequality (2.17) shows that $E_{q, \omega}(t)$ is positive and is bounded on every compact subinterval of $\left(\omega_{0}-\frac{1}{1-q}, \omega_{0}+\frac{1}{1-q}\right)$. Also we can see that

$$
\begin{aligned}
\left|E_{q, \omega}\left(h^{n}(t)\right)\right| & \leq \prod_{k=0}^{\infty}\left|1+q^{k+n}(t(1-q)-\omega)\right| \\
& \leq \prod_{k=0}^{\infty}\left[1+q^{k+n}(1-q)\left|t-\omega_{0}\right|\right] \\
& \leq \prod_{k=0}^{\infty}\left[1+q^{k+n}\right] \\
& \leq e^{\frac{1}{1-q}} .
\end{aligned}
$$


Therefore,

$$
\begin{aligned}
\left|D_{q, \omega}^{n} E_{q, \omega}(t)\right| & \leq q^{\frac{n(n-1)}{2}}\left|E_{q, \omega}\left(h^{n}(t)\right)\right| \\
& \leq q^{\frac{n(n-1)}{2}} e^{\frac{1}{1-q}} .
\end{aligned}
$$

By Theorem 2.10, the $q, \omega$-Taylor expansion of $E_{q, \omega}(t)$ at $a$ is given by

$$
E_{q, \omega}(t)=\sum_{k=0}^{\infty} \frac{q^{\frac{k(k-1)}{2}} E_{q, \omega}\left(h^{k}(a)\right)}{[k]_{q} !} H_{k}(t, a) .
$$

Since $D_{q, \omega}^{n} f\left(\omega_{0}\right)=q^{\frac{n(n-1)}{2}}$, the $q$, $\omega$-Taylor expansion of $E_{q, \omega}(t)$ at $\omega_{0}$ is given by

$$
E_{q, \omega}(t)=\sum_{k=0}^{\infty} \frac{q^{\frac{k(k-1)}{2}}}{[k]_{q} !}\left(t-\omega_{0}\right)^{k} .
$$

The series in (2.25) is uniformly convergent on every compact subinterval of $\left(\omega_{0}-\frac{1}{1-q}\right.$, $\left.\omega_{0}+\frac{1}{1-q}\right)$ and consequently $E_{q, \omega}(t)$ is continuous.

\section{Acknowledgements}

Authors are thankful to the learned referees for their valuable comments which improved the presentation of the paper.

\section{Funding}

Not applicable.

\section{Availability of data and materials}

The data and material in this paper are original.

\section{Competing interests}

The authors declare that they have no competing interests.

Authors' contributions

All authors read and approved the final manuscript.

\section{Author details}

'Department of Mathematics and Computer Science, Faculty of Science, Suez University, Suez, Egypt. ${ }^{2}$ Department of Mathematics, Faculty of Science, Cairo University, Giza, Egypt. ${ }^{3}$ Department of Mathematics, Faculty of Science, University of Jeddah, Jeddah, Saudi Arabia.

\section{Publisher's Note}

Springer Nature remains neutral with regard to jurisdictional claims in published maps and institutional affiliations.

Received: 10 July 2019 Accepted: 23 April 2020 Published online: 04 May 2020

\section{References}

1. Aldwoah, K.A.: Generalized time scales and associated difference equations. Ph.D. thesis, Cairo University (2009)

2. Annaby, M.H., Hamza, A.E., Aldwoah, K.A.: Hahn difference operator and associated Jackson-Norlund integrals. J. Optim. Theory Appl. 154, 133-153 (2012)

3. Annaby, M.H., Mansour, Z.S.: $q$-Taylor and interpolation series for Jackson q-difference operators. J. Math. Anal. Appl. $344,472-483(2008)$

4. Bird, M.T:: On generalizations of sum formulas of the Euler-Maclaurin type. Am. J. Math. 58, 487-503 (1936)

5. Birkhoff, G.D.: General theory of linear difference equations. Trans. Am. Math. Soc. 12, 243-284 (1911)

6. Brikshavana, T., Sitthiwirattham, T.: On fractional Hahn calculus. Adv. Differ. Equ. 2017, Article ID 354 (2017)

7. Cigler, J.: Elementare q-Identitäten. Publication de l'institute de recherche Mathématique avancée, 23-57 (1982)

8. Euler, L.: Introductio in Analysin Infinitorum (1748). Chapter VII

9. Gasper, G., Rahman, M.: Basic Hypergeometric Series. Cambridge University Press, Cambridge (2004)

10. Hahn, W. Über orthogonalpolynome, die q-differenzenlgleichungen genügen. Math. Nachr. 2, 4-34 (1949)

11. Jackson, F.H.: On q-functions and a certain difference operator. Trans. R. Soc. Edinb. 46, 253-281 (1908)

12. Jackson, F.H.: q-Form of Taylor's theorem. Messenger Math. 39, 62-64 (1909) 
13. Jackson, F.H.: On q-difference equations. Am. J. Math. 32, 305-314 (1910)

14. Jagerman, D.L.: Difference Equations with Applications to Queues. Marcel Dekker, New York (2000)

15. Jordan, C.: Calculus of Finite Differences. Chelsea, New York (1965)

16. Malinowska, A.B., Martins, N.: Generalized transversality conditions for the Hahn quantum variational calculus. Optimization 62, 323-344 (2013)

17. Malinowska, A.B., Torres, D.F.: The Hahn quantum variational calculus. J. Optim. Theory Appl. 147, 419-442 (2010)

18. Patanarapeelert, N., Brikshavana, T., Sitthiwirattham, T.: On nonlocal Dirichlet boundary value problems for sequential Caputo fractional Hahn integrodifference equations. Bound. Value Probl. 2018, Article ID 6 (2018)

19. Patanarapeelert, N., Sitthiwirattham, T.: Existence results for fractional Hahn difference and fractional Hahn integral boundary value problems. Discrete Dyn. Nat. Soc. 2017, Article ID 7895186 (2017)

20. Patanarapeelert, N., Sitthiwirattham, T.: On nonlocal Robin boundary value problems for Riemann-Liouville fractional Hahn integrodifference equation. Bound. Value Probl. 2018, Article ID 46 (2018)

21. Sitthiwirattham, T.: Nonlocal three-point boundary value problems for nonlinear second-order Hahn difference equations with two different q, $\omega$ derivatives. Adv. Differ. Equ. 2016, Article ID 116 (2016)

22. Wang, Y., Liu, Y., Hou, C.: New concepts of fractional Hahn's q, $\omega$-derivative of Riemann-Liouville type and Caputo type and applications. Adv. Differ. Equ. 2018, Article ID 292 (2018)

\section{Submit your manuscript to a SpringerOpen ${ }^{\circ}$ journal and benefit from:}

- Convenient online submission

- Rigorous peer review

Open access: articles freely available online

- High visibility within the field

- Retaining the copyright to your article

Submit your next manuscript at $\mathbf{s p r i n g e r o p e n . c o m ~}$ 